Original Contribution

\title{
THE INFLUENCE OF MANAGERS' EMOTIONAL INTELLIGENCE ON THEIR WORK PERFORMANCE IN BUSINESS ORGANIZATIONS (EMPIRICAL STUDY)
}

\author{
S. Masaldzhiyska* \\ Department of Industrial Business, Business Faculty, University of National and World Economy - \\ Sofia, Bulgaria
}

\begin{abstract}
In recent decades, the number of theoretical studies related to the psychological aspects of human resource management has increased. In the context of behavioral sciences, the orientation of scientists and researchers to the inner essence of individuals, to thought processes and the feelings of people at the workplace, and hence to the resulting work behavior, is completely natural. Work productivity stems from a certain type of employee behavior, and a number of factors influence this behavior in people's work activity. Emotional intelligence is one of the key factors influencing the work behavior of business organizations' leaders. This component encompasses abilities and skills that orient the behavior of managers in their work performance in a direction consistent with the organizational goals and the personal goals of each of these leaders.
\end{abstract}

Key words: emotional intelligence; work performance; mutual influence

\section{INTRODUCTION}

Modern business organizations operate in an economic environment which is dynamic, changing and largely unfavorable for them. This is particularly true for Bulgarian businesses. The strategic objective of ensuring the competitiveness and growth of industrial organizations in the country is heavily based on mechanisms for achieving a competitive advantage in human resources. Psychological and more specifically emotional intelligence and its impact on the work performance of human resources is a strand in human resource management with practical significance and utility. From a scientific point of view, a lot of research has been produced on this issue. There is scientific evidence of the significant influence of the emotional intelligence of human resources on their work performance. At the same time, there is a methodical and applied research gap concerning the above

\footnotetext{
*Correspondence to: Stefka Masaldzhiyska, Department of Industrial Business, Business faculty, University of National and World Economy - Sofia, Bulgaria
}

influence. In the present article, the focus is on key aspects of the impact of emotional intelligence on work performance studied through an own empirical survey, focused on managers in Bulgarian business organizations.

According to Singh, emotions are one of the warning systems of the human nature and are a complex state of the human mind that involves physiological changes on the one hand and psychological changes on the other. He defines intelligence as a person's cognitive ability, i.e. the ability to learn, think, and reason (1).

Emotional intelligence is defined by Mayer and Salovey as "the ability to perceive, evaluate and express emotions accurately; the ability to generate feelings that facilitate thought; the ability to understand emotions and to manage them in order to promote emotional and intellectual growth" (2). According to Petrides and Furnham emotional intelligence is the interconnection between emotionallydependent subjective perceptions and persuasions of the personality about sustainability and the personal qualities people use to manage emotions (3). Emotional intelligence, according to Golman, is a set of 
skills, including: the ability to motivate oneself, to persevere in difficult moments, to control one's impulses and to postpone the reward for one's efforts, to regulate one's moods and to prevent anxiety from interfering in one's thinking, to show empathy and to live with hope (4). Eketu and Ayondu define emotional intelligence as the ability to learn, understand and apply emotional knowledge and skills (5).

Emotional intelligence is vital to business, human work behavior, the quality of work, engagement and labor morale in general. Emotions, to a greater extent than intellectual abilities, determine the thinking of people and human interactions (6). Emotional intelligence is a set of capabilities that allow emotions to be properly used in decision-making and problem solving in situations which at first glance have a purely pragmatic and rational character. From this it can be concluded that intellectual and emotional skills, when combined, influence the thinking, behavior and work performance of employees. According to Paunov, mental structures that manage emotions and feelings have a greater impact on decision-making than logic and reason. Moreover, emotions have much more power to influence reason than vice versa (7). Employees who recognize their own emotions and know how to control them, while at the same time recognizing others' emotions, are productive, effective, work in a focused manner and achieve better results in their work.

Negative emotions experienced by employees seriously jeopardize the work process and may be an obstacle to increased performance at work. People working under stress think in a more limited scope and lose their imagination and creative thinking. Stress reduces their ability to sense and perceive the feelings, intentions and non-verbal messages coming from others. This leads to "internal closure", lack of empathy and insensitivity, which are signs of low emotional intelligence. On the other hand, employees with high emotional intelligence have the ability to immediately capture negative feelings and try to minimize their influence (8).

According to Quebbeman and Rozell, emotional intelligence has an impact on aggression at work. Although they have no empirical evidence, the authors conclude that people with high emotional intelligence and a strong positive outlook are more likely to respond to perceived injustices with adaptive and constructive behavior. These people are less likely to show aggression in the event of a conflict (9).

According to Fistolera, when basic emotional skills are present in the workplace, such as a harmonious perception of people's feelings and the ability to manage conflicts so as not to escalate, there are benefits to the work and the organization as a whole. Emotional intelligence helps employees in managing and expressing emotions so that they can achieve better results and cope more successfully in difficult situations (10).

Employees with a high level of emotional intelligence are more satisfied with their work and have positive attitudes towards clients and colleagues (11). Carmeli argues that emotional intelligence increases altruistic behavior, allowing employees to show a positive mood (12). Employees with a positive mood are more likely to provide help and support. When they feel emotionally strong, they show their commitment to the organization they work in (13). Emotional experiences at work affect the behavior and attitudes of employees (14).

Employees with a high level of emotional intelligence are able to understand organizational norms and rules and show a high degree of sensitivity to the work environment (15). Therefore, they are more inclined to show altruistic and polite behavior that is beneficial to the organization as a whole. The probability that such employees will experience high professional stress is lower. They respond with positive emotions to even the most undesirable situations. According to Wong and Law, staff with a high level of emotional intelligence can shape their reactions and are more effective in regulating emotions to achieve the desired emotional state (16). Quebbeman and Rozell theorize that employees with low levels of emotional intelligence are less able to limit negative emotions caused by negative work situations and are therefore more likely to react inappropriately, which can harm the organization or the other employees who work in it (9).

The ability of employees, and especially managers, to recognize and understand the feelings of others, as well as to respond to these feelings in an appropriate way, requires a certain level of "emotional education". People with a high level of emotional intelligence communicate well, share their intentions, 
ideas, goals, and are more positive, all of which are very important to the results that employees achieve in the workplace (17).

Emotional intelligence can have a significant impact on the business and team management in business organizations. An ever more significant portion of research links EI to creating strong relationships between employees and scientifically proves a positive correlation between emotional intelligence and successful organizational results $(18,19)$.

\section{MATERIALS AND METHODS}

From a methodological viewpoint, the determination of the degree of emotional intelligence of managers in business organizations encompasses the following stages and steps.

Stage I: Developing a questionnaire to determine the degree of emotional intelligence of managers in business organizations.

Stage II: Determining the degree of emotional intelligence.

Step 1: Calculating the estimates for the five components of emotional intelligence - SelfKnowledge, Self-Control, Motivation, Empathy, and Social Skills.

Step 2: Calculating the total estimate of emotional intelligence for each supervisor.

In this study, the determination of the level of emotional intelligence is based on a questionnaire developed by the author (20), which includes 50 questions. The questions in it refer only to the work process of managers in business organizations and measure the degree of emotional intelligence in the work environment.

Different aspects of the five components of emotional intelligence considered in the Golman theoretical model and Wood and Tolly's "Professional Emotional Intelligence Tests" have been taken into account in formulating the questions.

The processing of the results and the quantitative estimates of emotional intelligence for each and all respondents expressed as a percentage (\%) is introduced and processed using Microsoft Excel 2010 and the SPSS 20 statistical package.

The assessment of the performance of managers includes 8 steps.

Step 1: Defining Performance Indicators.

Step 2: Determining Degrees for Benchmarking.

Step 3: Determining coefficients for the importance of the Indicators.
Step 4: Identifying personal scores for the individual metrics.

Step 5: Identifying the maximum possible score for each metric.

Step 6: Determining the overall score for all performance indicators.

Step 7: Determining the sum of the maximum scores for all metrics.

Step 8: Calculating the coefficient that characterizes the overall job performance.

Evaluating the performance of managers is based on using the analytical approach and reaching quantitative estimates.

The range of indicators proposed in the article is as follows: 1. Result driven orientation;

2. Professional knowledge and skills;

3. Execution of the duties under the job description;

4. Ability to make decisions;

5. Motivation of the managed staff (or the team members);

6. Managing Conflict Situations;

7. Communication;

8. Building relationships;

9. Level of self-control;

10. Personal motivation;

11. Having empathy;

12. Leadership potential.

The first five indicators are defined as hard indicators and are merged into a Professional Skills scale.

The other seven benchmarking indicators are behavioral and are grouped into a Behavioral Skills scale.

The performance indicators for managers' performance are "decoded" using adequate and relevant indicators.

The benchmarks for assessing the indicators as well as the requirements for awarding the respective score of the indicators are determined.

It is imperative to determine the coefficients of the performance indicators in the performance appraisal in view of their "weight" in the overall assessment of the performance of managers.

The article does not readily refer to the algorithm for determining the personal scores and the overall assessment of the performance of managers, which are measured through a quantifiable quotient.

Establishing the values of the emotional intelligence of leaders and their performance 
are in their essence the two phases that precede the determination of the influence of the emotional intelligence of managers on their performance and the strength of that influence.

\section{RESULTS AND DISCUSSION}

The empirical study was conducted through a survey of 51 employees who hold senior positions in 5 business organizations.

The aim of the study presented in the article is to determine the influence of the emotional intelligence of these leaders on their performance in the selected organizations as well as the power of this influence.

\section{Tasks of the empirical research}

1. To analyze and evaluate the degree of emotional intelligence of managers and their performance in business organizations.

2. To study the impact of emotional intelligence on the general performance of managers in business organizations.

3. To investigate the impact of emotional intelligence on managers' performance by analyzing and assessing behavioral ("soft") indicators to assess their performance in business organizations.

\section{Hypotheses of the empirical research}

Hypothesis 1: The higher degree of emotional intelligence of managers is a prerequisite and a factor for their better overall performance.

Hypothesis 2: The higher degree of emotional intelligence of the managers has a strong positive influence on their behavior and the level of behavioral indicators in their work activity.

- Analysis and assessment of the level of emotional intelligence of managers in business organizations

Emotional Intelligence (EI) includes the following five components: self-knowledge, selfcontrol, motivation, empathy and social skills. Each of the components is represented through a three-level Likert Scale ("High Level", "Medium Level" and "Low Level"). Summarizing the scores of the five components for the high, middle and low levels (or degrees) of emotional intelligence of managers, three EI scales have been deduced: "High EI", "Medium EI" and "Low EI". Their values are expressed in relative shares $(\%)$.

The author has verified the reliability of the High EI, Medium EI and Low EI scales based on the calculated Cronbach's alpha coefficients. The results obtained are presented in Table 1.

Table 1. Efficiency ratios of emotional intelligence scales

\begin{tabular}{|c|c|c|r|r|}
\hline EI scales & $\begin{array}{c}\text { Number of } \\
\text { items }\end{array}$ & $\begin{array}{c}\text { Cronbach's } \\
\text { alpha }\end{array}$ & Mean & Std. Deviation \\
\hline High EI & 5 & 0.778 & 30.08 & 6.72 \\
\hline Medium EI & 5 & 0.534 & 13.75 & 4.12 \\
\hline Low EI & 5 & 0.731 & 6.18 & 4.25 \\
\hline
\end{tabular}

For the High EI scale, which includes five items (or components), the mean value of the scale is Mean $=30.08$ with standard deviation Std. Deviation $=6.72$. Cronbach's Alpha coefficient is equal to 0.778 . This coefficient is statistically significant with significance level Sig. $F=0.000<\alpha=0.05$. It is greater than 0.7 , therefore there is strong internal consistency between the items in the scale.

For the Medium EI scale, which includes the same five items, the average value of the scale is Mean $=13.75$ with standard deviation Std. Deviation $=4.12$. Cronbach's Alpha coefficient of is equal to 0.534. The ANOVA test indicates that it is a statistically significant coefficient, as Sig. $F=0.000<\alpha=0.05$. This coefficient is less than 0.7 , therefore there is a medium-to-moderate internal consistency between the items forming the scale.
For the Low EI scale, which also includes five items, the mean value of the scale is Mean = 6.18 with the standard deviation Std. Deviation $=4.25$. Cronbach's Alpha coefficient is equal to 0.731 . The ANOVA test shows that this coefficient is statistically significant, ie. there is consistency between the numbers, as Sig. F $=0.000<\alpha=0.05$. This coefficient is greater than 0.7 , therefore there is a strong internal consistency between the items in the scale.

Cronbach's Alpha coefficient is highest for the High EI scale, at 0.778 , followed by the Cronbach's Alpha coefficient for the Low EI scale, which equals 0.731 , and the smallest is Cronbach's Alpha coefficient of for the Medium EI scale - 0.534. The first and third coefficients have a high reliability level of greater than 0.7 while the second coefficient has an average reliability. 
Table 2 presents the descriptive statistics of the three EI scales, namely High EI, Medium EI and Low EI and it includes the following
MASALDZHIYSKA S.

values: Mean, Standard Error of Mean, Standard. Deviation, Minimum, Maximum, Skewness and Kurtosis.

Table 2. Descriptive statistics of the emotional intelligence scales

\begin{tabular}{|c|c|c|c|c|}
\hline \multicolumn{5}{|c|}{ Statistics } \\
\hline & & High EI $(\%)$ & Medium EI (\%) & Low EI (\%) \\
\hline \multirow{2}{*}{$\mathrm{N}$} & Valid & 51 & 51 & 51 \\
\hline & Missing & 0 & 0 & 0 \\
\hline \multicolumn{2}{|c|}{ Mean } & 60,16 & 27,49 & 12,35 \\
\hline \multicolumn{2}{|c|}{ Std. Error of Mean } & 1,882 & 1,153 & 1,190 \\
\hline \multicolumn{2}{|c|}{ Std. Deviation } & 13,439 & 8,235 & 8,501 \\
\hline \multicolumn{2}{|c|}{ Std. Error of Skewness } & ,333 & ,333 & ,333 \\
\hline \multicolumn{2}{|c|}{ Kurtosis } & 1,004 &,- 158 & 8,874 \\
\hline \multicolumn{2}{|c|}{ Std. Error of Kurtosis } &, 656 &, 656 &, 656 \\
\hline \multicolumn{2}{|c|}{ Minimum } & 20 & 14 & 2 \\
\hline \multicolumn{2}{|c|}{ Maximum } & 82 & 46 & 52 \\
\hline
\end{tabular}

The table shows that the highest share of the surveyed managers has a high level of emotional intelligence - $60.16 \%$. A medium level of emotional intelligence was exhibited by $27.49 \%$ and only $12.35 \%$ have a low level of emotional intelligence. This result justifies continuing the analysis by examining managers with a high level of emotional intelligence.

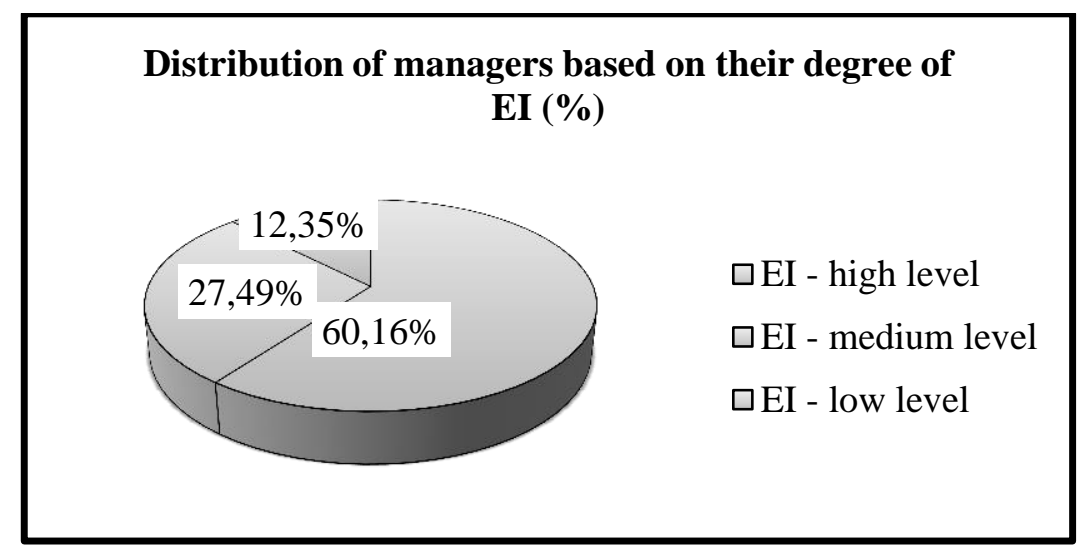

Figure 1. Distribution of managers based on their degree of emotional intelligence (\%)

The table below (Table 3) presents the distribution of leaders by their degree of emotional intelligence and its determinant components - self-knowledge, self-control, motivation, empathy and social skills.

Table 3. Distribution of leaders by their degree of emotional intelligence and its determinant components (\%)

\begin{tabular}{|c|c|c|c|}
\hline \multicolumn{4}{c|}{ Degree of emotional intelligence } \\
\hline Components & High EI & Medium EI & Low EI \\
\hline Self-knowledge & $40.8 \%$ & $36.9 \%$ & $22.4 \%$ \\
\hline Self-control & $46.3 \%$ & $36.9 \%$ & $16.9 \%$ \\
\hline Motivation & $70.4 \%$ & $22.7 \%$ & $6.9 \%$ \\
\hline Empathy & $68.0 \%$ & $23.1 \%$ & $8.8 \%$ \\
\hline Social skills & $75.3 \%$ & $17.8 \%$ & $6.9 \%$ \\
\hline Total: & $\mathbf{6 0 . 2 \%}$ & $\mathbf{2 7 . 4 \%}$ & $\mathbf{1 2 . 4 \%}$ \\
\hline
\end{tabular}


Comparing the relative shares of the components, in the High EI scale, social skills have the biggest relative share at $75.3 \%$, followed by motivation at $70.4 \%$ and empathy at $68.0 \%$ while self-control ranks fourth with $46.3 \%$ and self-knowledge comes last at $40.8 \%$.

In the Medium EI scale, self-awareness and self-control hold the highest relative share at $36.9 \%$ followed by empathy at $23.1 \%$, motivation at $22.7 \%$ and social skills at $17.8 \%$.

In the Low EI scale, self-knowledge ranks first with a relative share of $22.4 \%$, self-control is second at $16.9 \%$, empathy follows with $8.8 \%$, and motivation and social skills each have a $6.9 \%$ share.
- Exploring the impact of emotional intelligence on work performance

The following variables are considered:

- The independent variable (factor) is high emotional intelligence degree (EI high) of managers, measured as a percentage;

- the dependent variable (result) is the work performance (WP), measured as a percentage.

The study looks for a linear correlation pattern of the type:

$$
\hat{\mathrm{WP}}(\%)=b_{Q}+b_{l} \operatorname{EI} h i g h(\%)
$$

The following results were obtained:

Table 4. ANOVA test to determine correlational dependence

\begin{tabular}{|l|r|r|r|r|r|}
\hline \multicolumn{7}{|c|}{ ANOVA } \\
\hline & Sum of Squares & df & Mean Square & F & Sig. \\
\hline Regression & 1728,927 & 1 & 1728,927 & 25,124 &, 000 \\
\hline Residual & 3371,940 & 49 & 68,815 & & \\
\hline Total & 5100,867 & 50 & & & \\
\hline The independent variable is High emotional intelligence degree (\%). \\
\hline
\end{tabular}

The ANOVA test shows that between EI high $(\%)$ и $W P(\%)$ there is a linear dependence, as Sig. $F=0.000<\alpha=0.05$.

Table 5. Coefficients of the model

\begin{tabular}{|c|c|c|c|c|c|}
\hline \multicolumn{6}{|c|}{ Coefficients } \\
\hline & \multicolumn{2}{|c|}{ Unstandardized Coefficients } & \multirow{2}{*}{$\begin{array}{c}\begin{array}{c}\text { Standardized } \\
\text { Coefficients }\end{array} \\
\text { Beta } \\
\end{array}$} & \multirow[t]{2}{*}{$\mathrm{t}$} & \multirow[t]{2}{*}{ Sig. } \\
\hline & $\mathrm{B}$ & Std. Error & & & \\
\hline $\begin{array}{l}\text { High emotional } \\
\text { intelligence degree }(\%)\end{array}$ & ,438 & 087 &, 582 & 5,012 & ,000 \\
\hline (Constant) & 48,229 & 5,378 & & 8,968 &, 000 \\
\hline
\end{tabular}

Table 5 shows that the constant $b_{0}=48.229$. This coefficient is statistically significant because its significance level is Sig. $t=0.000<$ $\alpha=0.05$. The regression coefficient $b_{1}=0.438$ is also statistically significant because its level of significance Sig. $t=0.000<\alpha=0.05$.

Therefore, the linear correlation model obtained looks as below:

$$
\hat{\mathrm{WP}}(\%)=48.229+0.438 \mathrm{EI} \text { high }(\%)
$$

The regression coefficient $b_{1}=0.438$ is positive, which means that there is a direct dependence between the two dimensions observed, i.e. with an increase in the emotional intelligence EI high of managers (\%) their work performance coefficient $(\%)$ also grows. The regression coefficient represents the rate of growth, i.e. $1 \%$ growth of EI high (\%), results in a $0.438 \%$ increase of the work performance (WP) coefficient.

Table 6. Coefficients $R, R^{2}$ and Std. Error of the Estimate

\begin{tabular}{|r|c|c|c|}
\hline \multicolumn{5}{|c|}{ Model Summary } \\
\hline R & R Square & Adjusted R Square & Std. Error of the Estimate \\
\hline, 582 &, 339 &, 325 & 8,295 \\
\hline \multicolumn{3}{|c|}{ The independent variable is High emotional intelligence degree (\%). } \\
\hline
\end{tabular}


The correlation coefficient which expresses the strength of correlation dependence is $\mathrm{R}$ $=0.582<0.7$. It shows that there is a medium correlation between EI high (\%) и WP (\%).

The coefficient of determination is $R^{2}=$ $0.339=33.9 \%$. It shows that $33.9 \%$ of the variation in work performance is determined by the variation of the EI high (\%) factor. The Standard error of the estimate is Std. Error of the Estimate $=8.295(\%)$.

The linear dependency WP $(\%)=48.229+$ 0.438 EIhigh $(\%)$ shows that the increase in emotional intelligence is a prerequisite for better performance, which confirms Hypothesis 1.

- Study of the impact of managers' emotional intelligence on their performance
MASALDZHIYSKA S.

through analysis and assessment of behavioral performance indicators.

- The independent variable (factor) is high emotional intelligence degree (EI high) of managers, measured as a percentage;

- The dependent variables (result) are behavioral skills (BS) of managers, measured as a percentage.

The study looks for a linear correlation pattern of the type:

$\wedge$

$\mathrm{BS}(\%)=b_{0}+b_{l} \operatorname{EI} h i g h(\%)$

The following variables are considered:

Table 7. ANOVA test to determine correlation dependence

\begin{tabular}{|l|r|r|r|r|r|}
\hline \multicolumn{7}{|c|}{ ANOVA } \\
\hline & Sum of Squares & df & Mean Square & F & Sig. \\
\hline Regression & 2750,753 & 1 & 2750,753 & 25,205 &, 000 \\
\hline Residual & 5347,667 & 49 & 109,136 & & \\
\hline Total & 8098,420 & 50 & & & \\
\hline The independent variable is High emotional intelligence degree (\%). \\
\hline
\end{tabular}

The ANOVA test shows that there is a linear correlation between EI high (\%) and BS (\%), as Sig. $F=0.000<\alpha=0.05$.

Table 8. Coefficients of the model

\begin{tabular}{|c|c|c|c|c|c|}
\hline \multicolumn{6}{|c|}{ Coefficients } \\
\hline & \multicolumn{2}{|c|}{$\begin{array}{l}\text { Unstandardized } \\
\text { Coefficients }\end{array}$} & $\begin{array}{l}\text { Standardized } \\
\text { Coefficients }\end{array}$ & $\mathrm{t}$ & Sig. \\
\hline & B & Std. Error & Beta & & \\
\hline $\begin{array}{l}\text { High emotional intelligence } \\
\text { degree }(\%)\end{array}$ & ,552 & , 110 & 583 & 5,020 & 000, \\
\hline (Constant) & 37,985 & 6,773 & & 5,608 & ,000 \\
\hline
\end{tabular}

Table 8 shows that the constant $b_{0}=37.985$. This coefficient is statistically significant because its significance level is Sig. $t=0.000<$ $\alpha=0.05$. The regression coefficient $b_{1}=0.552$ is also statistically significant because its level of significance is Sig. $t=0.000<\alpha=0.05$.

Therefore, the linear correlation model obtained looks as below:
The regression coefficient $b_{1}=0.552438$ is positive, which means that there is a direct dependence between the two values observed, i.e. with an increase in the emotional intelligence EI high of managers (\%) their behavioral skills coefficient (\%) also grows. The regression coefficient represents the rate of growth, i.e. a $1 \%$ growth of EI high (\%), results in a $0.552 \%$ increase of the behavioral skills (BS) coefficient.

BS $(\%)=37.985+0.552$ EIhigh $(\%)$ 
Table 9. Coefficients $R, R^{2}$ and Std. Error of the Estimate

\begin{tabular}{|c|c|c|c|}
\hline \multicolumn{5}{|c|}{ Model Summary } \\
\hline $\mathrm{R}$ & R Square & Adjusted R Square & $\begin{array}{c}\text { Std. Error of the } \\
\text { Estimate }\end{array}$ \\
\hline \multicolumn{2}{|c|}{, 583} &, 340 &, 326 \\
\hline
\end{tabular}

The correlation coefficient is $\mathrm{R}=0.583<0.7$. It shows that there is a medium correlation between EI high (\%) and BS (\%).

The coefficient of determination is $R^{2}=$ $0.340=34.0 \%$. It shows that $34.0 \%$ of the variation in behavioral skills is determined by the variation of the EI high (\%) factor.

The Standard error of the model is Std. Error of the Estimate $=10.447(\%)$.

The linear dependency BS $(\%)=37.985+$ 0.552 EIhigh $(\%)$ shows that the increase in emotional intelligence has a strongly positive influence on behavioral performance indicators for managers, which confirms Hypothesis 2.

\section{CONCLUSION}

This empirical study, presented in a synthesized manner, is based on the author's self-developed research methodology and is part of a richer study. It gives grounds to draw the following conclusions:

- The study has focused on managers with a high level of emotional intelligence. The specifically chosen five components of emotional intelligence and their contribution to the overall assessment of emotional intelligence enable us to realistically highlight good and bad practices based on the following approach: high scores on the individual components of emotional intelligence are good practices; low scores represent bad practices. The approach taken has utility and can be used in two aspects: it targets the necessary improvements in the low-rated components of the emotional intelligence of the managers and it opens up opportunities to transfer and use experience in validating capabilities and skills within the range of emotional intelligence where there are highly rated components.

- The two scales of the indicators for the performance of the managers, respectively the Professional Skills scale and the Behavior Skills scale, and the established average values of the two scales show a higher average for the first scale. Higher rated hard indicators from the first scale are to a certain extent attributable to their assigned higher significance ratios given by those surveyed. A second important argument in this regard is related to the wellestablished belief in managers that their performance depends (and is assessed) mainly based on hard and formalized indicators, and to a lesser extent on indicators that characterize their behavior in the work activity.

- The specific values of the indicators on the two scales as ordered in descending order reveal the good and bad practices in the managers' performance. For example, for the "Professional Skills" scale, the average score for the "Professional Knowledge and Skill" (10.35) is highest, while the "Employee Motivation" indicator is the lowest (7.69). For the "Behavioral Skills" scale, the average score for the "Communication" indicator (10.75) is highest, and Leadership Potential (6.16) and "Building Relationships" (6.24) are at the bottom. The low values of these indicators in the two scales represent real problem areas in the work performance of the surveyed managers. Opportunities to raise the low values of these indicators should be sought and utilized on the basis of managers' selfassessment of weaknesses and shortcomings in each indicator.

- This article establishes the influence of the emotional intelligence of managers on their performance in two main directions. In the first direction, a multifactorial linear regression model is used to determine the influence of the EI of managers on their overall performance. There is evidence which leads to the exclusion from the study of managers exhibiting two of the factor variables: Medium EI and Low EI, from there, the study is based on a one-factor linear correlation model, covering only the influence of High EI of senior executives on their performance. The results obtained confirm the existence of a direct dependence between the emotional intelligence of managers and their performance, i.e. with the increase of the emotional intelligence of the leaders, the coefficient of their performance rises - there 
are improvements in the work performance of the managers that reflect the strength of the above influence.

- The study of the influence of high EI on the "Behavioral Skills" scale (or behavioral performance indicators) confirms the direct relationship between the two indicators; it provides evidence that the coefficient of behavioral skills (indicators) grows steadily with the increase in the EI (\%) of managers. In this respect, the influence of emotional intelligence is most significant when applied to the behavioral indicators in the assessment of their performance.

- The statistical tools used are instrumental for the results obtained on the influence of managers' emotional intelligence on their performance. The above-mentioned tools are indispensable for application in the practice of Bulgarian organizations in order to discern the above explained influence and reveal problem areas in order to overcome them. It can be utilized (including through training) by managers and specialists in human resources management in organizations.

- The topic presented in the article on the impact of emotional intelligence on work performance, for understandable reasons, is directed at key figures and individuals from business organizations such as managers in the leadership hierarchy. At the same time, future research can be targeted at:

- representatives of the other two categories of staff in business organizations: specialists and workers;

- key representatives of the specialist category, i.e. specialist employees with a significant contribution to the organization or expected significant contribution;

-enriching research strands where it would be possible to investigate and establish the influence of emotional intelligence on work performance, etc.

In summary, it can be concluded that the studied issues and the achieved results are a step in enriching the field of human resource management in business organizations and in particular in its psychological direction. This scientific methodological and practical enrichment has a clear economic dimension in the quality of leadership in business organizations and its impact on the achieved organizational goals.

\section{REFERENCES}

1. Singh, D., Emotional intelligence at work A professional guide (3rd Ed.), Sage Publications, New Delhi, p.30, 2006.

2. Salovey, P., Slutyer, D., Emotional Development and Emotional Intelligence: Educational Implications, New York: Basic Books, p.10, 1997.

3. Petrides, K. and Furnham A. (2000). On the dimensional structure of emotional intelligence, Personality and Individual Differences, 29: 313 - 320, 2000.

4. Goleman, D., Emotional Intelligence, EastWest, Sofia, 2011, p.57.

5. Eketu, C. and Ayondu, O., Devolving the Thought on Workplace Emotions for Gainful Research: A Theoretical Reflection. Journal of Organisation \& Human Behaviour, 6: 1-14, 2017.

6. Usheva, M., The Emotional Index - a Determining Factor for the Development of Innovative Leadership in Contemporary Management, Governance and Sustainable Development 1, 32: 140-145, 2012.

7. Paunov, M., Emotions, Organizational Behavior and Human Resources, Avangard Prima, Sofia, p. 212, 2010.

8. Simeonova, D., Raise emotionally intelligent employees, 2006, Available at https://www.karieri.bg/management/26152 8_vuzpitavaite_emocionalno_inteligentni_s lujiteli/.

9. Quebbeman, A. and Rozell, E., Emotional intelligence and dispositional affectivity as moderators of workplace aggression: The impact of behavior choice. Human Resource Management Review, 12: 125143, 2002.

10.Fistolera, P., Mastering emotions or how emotional intelligence interferes and helps in the corporate world, 2014, Available at http://www.capital.bg/politika_i_ikonomika /bulgaria/2014/05/21/2304638_ovladiavane nna_emociite/.

11.Day, A. and Carroll, S., Using an abilitybased measure of emotional intelligence to predict individual performance, group performance, and group citizenship behaviours. Personality and Individual Differences, 36: 1443-1458, 2004.

12.Carmeli, A., The relationship between emotional intelligence and work attitudes, behavior and outcomes: An examination among senior managers. Journal of Managerial Psychology, 18: 788-813, 2003.

13. Anari, N., Teachers: emotional intelligence, job satisfaction, and organizational 
commitment. Journal of Workplace Learning, 24: 256-269, 2012.

14.Jam, F., Sheikh, R., Iqbal, H., Zaidi, B., Anis, Y. and Muzaffar, M., Combined effects of perception of politics and political skill on employee job outcomes. African Journal of Business Management, 5: 9896-9904, 2011.

15.Carmeli, A., and Josman, Z., The relationship among emotional intelligence, task performance and organizational citizenship behaviors. Human Performance, 19: 403-409, 2006.

16. Wong, C. and Law, K., The effect of leader and follower emotional intelligence on performance and attitude: an exploratory study. The Leadership Quarterly, 13: 243274, 2002.

17.Arfara, Ch. and Samanta, I., The impact of emotional intelligence on improving teamworking: the case of Public Sector (National Centre for Public Administration
MASALDZHIYSKA S.

and Local Government - N.C.P.A.L.G.), 3rd International Conference on New Challenges in Management and Organization: Organization and Leadership, Dubai, UAE, Procedia - Social and Behavioral Sciences 230, p. 167 - 175, 2016.

18.Clarke, N., Emotional intelligence abilities and their relationships with team processes. Team Performance Management, 16: 6-32, 2010.

19.Rozell, E. and Scroggins, W., How much is too much? The role of emotional intelligence in self- managed work team satisfaction and group processes. Team Peroformance Management, 16: 33-49, 2010.

20.Masaldzhiyska, S., Influence of managers' emotional intelligence on their work performance in business organizations. Dissertation, Sofia: UNWE, 2018. 